\title{
Safety-Critical Kinematic Control of Robotic Systems
}

\author{
Andrew Singletary ${ }^{1}$, Shishir Kolathaya ${ }^{2}$, and Aaron D. Ames ${ }^{1}$
}

\begin{abstract}
Over the decades, kinematic controllers have proven to be practically useful for applications like set-point and trajectory tracking in robotic systems. To this end, we formulate a novel safety-critical paradigm by extending the methodology of control barrier functions (CBFs) to kinematic equations governing robotic systems. We demonstrate a purely kinematic implementation of a velocity-based $\mathrm{CBF}$, and subsequently introduce a formulation that guarantees safety at the level of dynamics. This is achieved through a new form of CBFs that incorporate kinetic energy with the classical forms, thereby minimizing model dependence and conservativeness. The approach is then extended to underactuated systems. This method and the purely kinematic implementation are demonstrated in simulation on two robotic platforms: a 6-DOF robotic manipulator, and a cart-pole system.
\end{abstract}

Index Terms-Robotics, Robust control, Safety-critical control

\section{INTRODUCTION}

$\mathbf{K}^{\mathrm{s}}$ INEMATIC control provides a powerful method for achieving desired behaviors on a large class of robotic systems [2], [3], [4]. Ensuring safety for these kinematic systems is widely researched area. Artificial potential field methods were formulated as a way to reach goal positions while avoiding obstacles utilizing an attractive force from the goal and a repulsive force from the obstacles [5]. In [6], the authors improve upon this idea by constructing the problem as a quadratic progarm $(\mathrm{QP})$, where the objective is to track the desired goal subject to geometric constraints on the velocities to prevent collisions. While this work is effective in practice, and has been extended to multi-objective task structures [7], it can be made more general and more formal through control barrier functions (CBFs) [8].

CBFs provide a framework for formally incorporating general safety constraints into quadratic programs. This was first applied to adaptive cruise control, and has since been utilized in a variety of application domains: automotive safety [9], robotics [10], [11] and multi-agent systems [12], [13]. See [14] for a recent survey. While CBFs can be implemented in a purely kinematic fashion for robotic systems [15], as will be demonstrated in this work, it only guarantees safety kinematically, like the above methods, not for the true underlying dynamical system. However, when the dynamics are used, it becomes heavily model-dependent, and the safety guarantees depend on the validity of the model.

Recently, energy-based reciprocal control barrier functions were introduced [16] as a means to provide robust safety

\footnotetext{
${ }^{1}$ Mechanical and Civil Engineering, Caltech, Pasadena, CA, USA, 91125 \{ames, asinglet\}@ealtech.edu

${ }^{2}$ Cyber Physical Systems and Computer Science and Automation, IISc, Bengaluru, KA, India, 560012. \{shishirk\}@iisc.ac.in
}

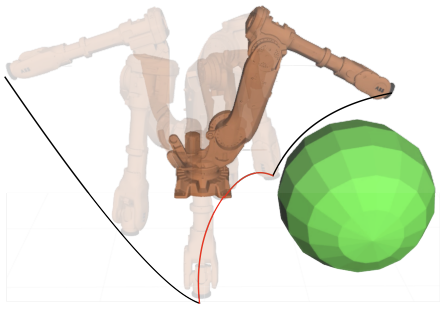

Fig. 1. A 6-DOF manipulator safely avoiding an obstacle with energybased control barrier function. The CBF intervention is shown in red. A video can be found at [1].

guarantees for fully-actuated robotic platforms with model uncertainty. This was done by utilizing bounds on the inertia and Coriolis-centrifugal matrices, as well as the gravity vector, and providing safety guarantees for the worst-case scenario. While the resulting QP formulation yielded robustness in safety, it does not have well-defined behavior on the boundary of the set and outside of it, making it difficult to implement in practice.

In this paper, an alternative formulation for the energybased CBFs is introduced for zeroing control barrier functions, which are well defined on the boundary and exterior of the set. Using this formulation, we modify the traditional torque-based formulation into a kinematic control problem, and showcase several simplifications that can be made to reduce model dependence. The resulting formulation allows for formal safety guarantees at the dynamical system level, while allowing for simple implementation with kinematic controllers. This analysis is then extended to the class of underactuated robotic systems. The results are demonstrated in a 6-DOF manipulator and a cart-pole system (see Figs. 1 and 4), wherein different levels of uncertainties are incorporated and safety-critical kinematic control laws are applied.

This paper is structured as follows. Section II provides the necessary background on CBFs. Section III demonstrates safety-critical velocity control of purely kinematic systems, with no regard for the underlying dynamics of the system. In Section IV, we begin with the formulation of an energybased CBF that guarantees the safety of a robotic system at the dynamics level. Then, this formulation is modified to guarantee safety of the dynamical system for kinematic control inputs, in this case a desired velocity command. The results are demonstrated in simulation on a 6 DOF robotic manipulator, and a comparison is made to the purely kinematic case. Finally, in Section V, the underactuated case is explored, and the method is demonstrated with a simulation of a cartpole system. 


\section{BACKGROUND}

Consider a nonlinear control system in affine form:

$$
\dot{x}=f(x)+g(x) u
$$

where $x \in \mathcal{D} \subset \mathbb{R}^{n}$ is the state, and $u \in U \subseteq \mathbb{R}^{m}$ the input. Assume that the functions $f: \mathbb{R}^{n} \rightarrow \mathbb{R}^{n}$ and $g: \mathbb{R}^{n} \rightarrow$ $\mathbb{R}^{n \times m}$ are continuously differentiable. We are interested in safety defined as the forward invariance of a set $\mathcal{S} \subset \mathcal{D}$. In particular, given a Lipschitz continuous control law $u=k(x)$, the resulting closed loop system $\dot{x}=f_{\mathrm{cl}}(x)=f(x)+g(x) k(x)$ yields a solution $x(t)$, with initial condition $x(0)=x_{0}$. The system is safe with respect to the controller $u=k(x)$ if:

$$
\forall x_{0} \in \mathcal{S} \quad \Rightarrow \quad x(t) \in \mathcal{S} \forall t \geq 0 .
$$

Definition 1 ([8]). Let $\mathcal{S} \subset \mathcal{D} \subset \mathbb{R}^{n}$ be the 0 -superlevel set of a continuously differentiable function $h: \mathcal{D} \rightarrow \mathbb{R}$ :

$$
\begin{aligned}
\mathcal{S} & =\left\{x \in \mathbb{R}^{n}: h(x) \geq 0\right\}, \\
\partial \mathcal{S} & =\left\{x \in \mathbb{R}^{n}: h(x)=0\right\}, \\
\operatorname{Int}(\mathcal{S}) & =\left\{x \in \mathbb{R}^{n}: h(x)>0\right\} .
\end{aligned}
$$

Then $h$ is a control barrier function (CBF) if $\frac{\partial h}{\partial x}(x) \neq 0$ for all $x \in \partial \mathcal{S}$ and there exists an extended class $\mathcal{K}$ function ([8, Definition 2]) $\alpha$ such that for the control system (1) and for all $x \in \mathcal{S}$ :

$$
\sup _{u \in U}[\underbrace{L_{f} h(x)+L_{g} h(x) u}_{\dot{h}(x, u)}] \geq-\alpha(h(x)),
$$

where $L_{f} h(x)=\frac{\partial h}{\partial x} f(x)$ and $L_{g} h(x)=\frac{\partial h}{\partial x} g(x)$. We say that $h$ is a control barrier function (CBF) on $\mathcal{S}$ if (2) holds for all $x \in \mathcal{S}$ (but not necessarily on all of $D$ ).

The main result with regard to control barrier functions is that the existence of a control barrier function implies that the control system is safe:

Theorem 1 ([8]). Let $\mathcal{S} \subset \mathbb{R}^{n}$ be a set defined as the superlevel set of a continuously differentiable function $h$ : $\mathcal{D} \subset \mathbb{R}^{n} \rightarrow \mathbb{R}$. If $h$ is a control barrier function $(C B F)$ on $\mathcal{S}$, then any Lipschitz continuous controller satisfying: $\dot{h}(x, u(x))=L_{f} h(x)+L_{g} h(x) u(x) \geq-\alpha(h(x))$ renders the set $\mathcal{S}$ safe for the system (1).

Controller Synthesis. The main idea with barrier functions is to use them as safety filters which take in a desired control input, $u_{\text {des }}(x, t)$, and modify this input in a minimal way so as to guarantee safety. This can be formalized as a Quadratic Program $(\mathrm{QP})$ :

$$
\begin{aligned}
u^{*}(x)=\underset{u \in U \subseteq \mathbb{R}^{m}}{\operatorname{argmin}} & \left\|u-u_{\mathrm{des}}(x, t)\right\|^{2} \quad(\text { CBF-QP) } \\
\text { s.t. } & L_{f} h(x)+L_{g} h(x) u \geq-\alpha(h(x)) .
\end{aligned}
$$

This controller has an explicit solution as noted by the following lemma.

Lemma 1. Let $h$ be a control barrier function for the control system (1) and assume that $U=\mathbb{R}^{m}$. Then the explicit solution to the $Q P(\mathrm{CBF}-\mathrm{QP})$ is given by:

$$
u^{*}(x, t)=u_{\mathrm{des}}(x, t)+u_{\text {safe }}(x, t),
$$

where $u_{\text {safe }}$ minimally modifies $u_{\mathrm{des}}$ depending on if the nominal controller keeps the system safe, i.e., the sign of $\Psi\left(x, t ; u_{\mathrm{des}}\right):=\dot{h}\left(x, u_{\mathrm{des}}(x, t)\right)+\alpha(h(x))$, according to:

$u_{\text {safe }}(x, t)= \begin{cases}-\frac{L_{g} h(x)^{T}}{L_{g} h(x) L_{g} h(x)^{T}} \Psi\left(x, t ; u_{\mathrm{des}}\right) & \text { if } \Psi\left(x, t ; u_{\mathrm{des}}\right)<0 \\ 0 & \text { if } \Psi\left(x, t ; u_{\mathrm{des}}\right) \geq 0\end{cases}$

Proof. In [17], an explicit form for (CBF-QP) was found using the KKT conditions when $u_{\text {des }}(x, t)=0$. The same proof with a modified cost yields the desired result. Specifically, the dualprimal feasibility and complementary slackness conditions remain unchanged. Following [17, Proof of Theorem 8], the stationary condition becomes: $u^{*}(x, t)=\mu(x) L_{g} h(x)^{T}+$ $u_{\text {des }}(x, t)$, where $\mu$ comes from the KKT conditions. This results in the closed form solution. Finally, safety is guaranteed from Theorem 1.

\section{SAFETY-CRiticAl Kinematic CONTROL}

In this section, we consider safety-critical kinematic control. We provide an example of velocity-based kinematic control of a robotic manipulator, and analyze its ability to maintain safety. We are interested in kinematic mappings of the form: $x=y(q)$ where $q \in Q \subset \mathbb{R}^{k}, x \in \mathcal{D} \subset \mathbb{R}^{n}$ and thus $y$ : $Q \rightarrow \mathcal{D}$. Here, we assume that $k \geq n$, i.e., that there are more degrees of freedom than tasks. Here $x$ is the vector of "outputs" or "task" variables, i.e., a vector of elements which we wish to control, and $q$ is a vector consisting of the systems configuration, e.g., angles of the robotic system. The evolution of the task variables is therefore given by:

$$
\dot{x}=J_{y}(q) \dot{q} .
$$

In kinematic control, we view $\dot{q}$ as the input to the system. Specifically, we wish to determine a feedback control law: $\dot{q}=K(q, t)$ that achieves the desired properties.

Kinematic Trajectory Tracking. Suppose that we have a desired trajectory $x_{d}(t)$ for the task vector. The goal is to track this trajectory, i.e., for $e(t)=x(t)-x_{d}(t) \rightarrow 0$ with $x(t)$ satisfying (5). Differentiating this yields:

$$
\dot{e}=J_{y}(q) \dot{q}-\dot{x}_{d}(t) .
$$

Therefore, for $\gamma>0$, if we choose $\dot{q}$ such that $J_{y}(q) \dot{q}=$ $\dot{x}_{d}(t)-\gamma e$, we have $\dot{e}=-\gamma e \Rightarrow e(t)=\exp (-\gamma t) e(0)$. As a result, if we wish to track a trajectory, we can pick:

$$
\dot{q}(x, t)=J_{y}(q)^{\dagger}\left(\dot{x}_{d}(t)-\lambda e\right),
$$

with $J_{y}(q)^{\dagger}=J_{y}(q)^{T}\left(J_{y}(q) J_{y}(q)^{T}\right)^{-1}$, the Moore-Penrose (right) pseudoinverse, assumed to be well defined.

Safety-Critical Control. Equipped with $\dot{q}$, which will now serve as the desired (potentially unsafe) input $\dot{q}_{\text {des }}$, we can now impose safety. We have the following.

Lemma 2. Consider a kinematic safety constraint $h: Q \subset$ $\mathbb{R}^{k} \rightarrow \mathbb{R}$ and the corresponding safe set $\mathcal{S}=\{q \in$ $Q: h(q) \geq 0\}$ defined as the 0-superlevel set of $h$. If $J_{h}(q) \neq 0$, then the following velocity based controller:

$$
\dot{q}^{*}(q, t)=\underset{\dot{q} \in \mathbb{R}^{k}}{\operatorname{argmin}}\left\|\dot{q}-J_{y}(q)^{\dagger}\left(\dot{x}_{d}(t)-\lambda\left(y(q)-x_{d}(t)\right)\right)\right\|^{2}
$$




$$
\text { s.t. } \dot{h}(q, \dot{q})=J_{h}(q) \dot{q} \geq-\alpha(h(q)),
$$

ensures safety, i.e., $\mathcal{S}$ is forward invariant. Moreover, this has a closed form solution given by

$$
\dot{q}^{*}(x, t)=\dot{q}_{\mathrm{des}}(q, t)+ \begin{cases}-J_{h}(x)^{\dagger} \Psi\left(q, t ; \dot{q}_{\mathrm{des}}\right) & \text { if } \Psi\left(q, t ; \dot{q}_{\mathrm{des}}\right)<0 \\ 0 & \text { if } \Psi\left(q, t ; \dot{q}_{\mathrm{des}}\right) \geq 0\end{cases}
$$

where $\Psi\left(x, t ; \dot{q}_{\mathrm{des}}\right)=J_{h}(q) \dot{q}_{\mathrm{des}}(q, t)+\alpha(h(q))$.

Therefore, the controller (8) utilizes $\dot{q}_{\text {des }}$ whenever it is safe, i.e., when $\Psi\left(q, t ; \dot{q}_{\text {des }}\right) \geq 0$. Conversely, in the case when $\dot{q}_{\text {des }}$ is unsafe the controller takes over and enforces $\dot{h}=J_{h}(q) \dot{q}^{*}(q, t)=-\alpha(h)$ until $\dot{q}_{\text {des }}$ is safe again.

Example 1 (Manipulator Obstacle Avoidance). Consider a 6-DOF industrial manipulator (see Fig. 1) attempting to track a desired trajectory $x_{d}(t)$ using the desired velocity given in (6) with its end-effector. Note that CBFs have been successfully applied to robot manipulators in [10], [11], [15] via kinematic control. Suppose that the manipulator needs to complete this trajectory while avoiding an obstacle located at $\left(x_{0}, y_{0}, z_{0}\right)$. Thus, in the set $\mathcal{S}=\{q \mid h(q) \geq 0\}$, the end-effector must be at least a distance $d$ from the obstacle. A control barrier function representing this safety constraint is

$$
h(x)=\left(x-x_{0}\right)^{2}+\left(y-y_{0}\right)^{2}+\left(z-z_{0}\right)^{2}-d^{2} .
$$

By substituting this into (7) or (8), we obtain the results shown in Figure 2. Since this CBF does not take into account the system dynamics or the tracking ability of the low-level controller, safety is not guaranteed, but it can be achieved by proper choice of $\alpha$. In this case, with scalar multiple $\alpha \in$ $[0.5,1]$, the obstacle is avoided, but not for $\alpha \in[2,3]$.

\section{From Kinematics to Dynamics}

We now wish to establish the main result of this paper: that guarantees safety for the dynamics of a robotic system. To do this, we first introduce an alternative formulation of the energy-based CBFs shown in [16] for robotic systems. We consider Euler-Lagrangian dynamics of the form:

$$
D(q) \ddot{q}+C(q, \dot{q}) \dot{q}+G(q)=B u
$$

where $B \in \mathbb{R}^{k \times m}$ is the actuation matrix, $D, C, G$ are the inertia, Coriolis-centrifugal and gravity matrices respectively of appropriate dimensions. We assume $m \leq k$, wherein $m=$ $k$ with $B$ invertible corresponds to full actuation. From the

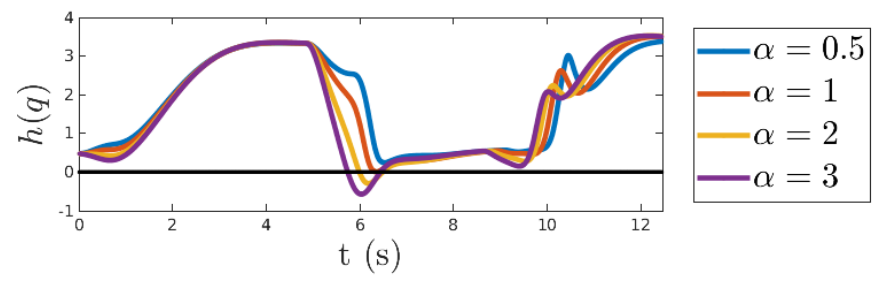

Fig. 2. Velocity-based kinematic barrier function on the $6 \mathrm{DOF}$ manipulator. Safety depends on choice of $\alpha$. The times to complete these tasks are shown in the video [1] and listed in Fig. 3. equations of motion, we can obtain a control system of the form (1). We will first discuss the fully actuated case, and the underactuated case will be discussed in Section V.

Energy-based Safety Constraints. We begin by formulating a safety-critical controller for fully actuated robotic systems given kinematic safety constraints - thus bridging the divide from kinematic to dynamics. This will be achieved via a "dynamically consistent" extension to the desired safe set. This is similar to the extensions shown in [14, Section IV], [18] for higher relative degree systems, but leverages the kinetic energy of the system. Specifically, to dynamically extend the CBF, we note that the inertia matrix, $D(q)$ is a symmetric positive definite matrix, $D(q)=D(q)^{T} \succ 0$, and thus:

$$
\lambda_{\min }(D(q))\|q\|^{2} \leq q^{T} D(q) q \leq \lambda_{\max }(D(q))\|q\|^{2}
$$

where $\lambda_{\min }$ and $\lambda_{\max }$ are the maximum and minimum eigenvalues (which are dependent on $q$ ) of $D(q)$ which are necessarily positive due to the positive definite nature of $D(q)$.

Definition 2. Given a kinematic safety constraint expressed as a function $h: Q \subset \mathbb{R}^{k} \rightarrow \mathbb{R}$ only dependent on $q$, and the corresponding safe set: $\mathcal{S}=\left\{(q, \dot{q}) \in Q \times \mathbb{R}^{k}: h(q) \geq 0\right\}$, the associated energy-based safety constraint is defined as:

$$
h_{D}(q, \dot{q}):=-\frac{1}{2} \dot{q}^{T} D(q) \dot{q}+\alpha_{e} h(q) \geq 0
$$

with $\alpha_{e}>0$. The corresponding energy-based safe set is:

$$
\mathcal{S}_{D}:=\left\{(q, \dot{q}) \in Q \times \mathbb{R}^{k}: h_{D}(q, \dot{q}) \geq 0\right\} .
$$

This construction is similar to the augmentation of kinetic energy in [16] for reciprocal control barrier functions. While the reciprocal formulation has the advantage of having no added conservatism, due to the set remaining unchanged, it does not have well-defined behavior on the boundary of the set and outside of it, making it less popular for implementation. In fact, we now will show that the energy based constraint in Definition 2 is a valid (zeroing) control barrier function (CBF), thereby allowing for a new class of QPs that guarantee safety. First, we establish the relationship between $\mathcal{S}_{D}$ and $\mathcal{S}$.

Proposition 1. Consider a kinematic safety constraint, $h$ : $Q \subset \mathbb{R}^{k} \rightarrow \mathbb{R}$, with corresponding safe set $\mathcal{S}$, and the associated energy-based safety constraint, $h_{D}$, as given in Definition 2 with corresponding safe set $\mathcal{S}_{D}$. Then

$$
\text { (i) } \mathcal{S}_{D} \subset \mathcal{S}, \quad \text { (ii) } \operatorname{Int}(\mathcal{S}) \subset \lim _{\alpha_{e} \rightarrow \infty} \mathcal{S}_{D} \subset \mathcal{S} \text {. }
$$

Proof. To establish (i), we simply note that

$\mathcal{S}_{D} \subset\left\{(q, \dot{q}) \in Q \times \mathbb{R}^{k}: h(q) \geq \frac{1}{2} \frac{\lambda_{\min }(D(q))}{\alpha_{e}}\|\dot{q}\|^{2} \geq 0\right\} \subset \mathcal{S}$.

To establish (ii), we first note that

$$
S_{D}\left(\alpha_{e}\right)=\left\{(q, \dot{q}) \in Q \times \mathbb{R}^{k}: h(q) \geq \frac{\frac{1}{2} \dot{q}^{T} D(q) \dot{q}}{\alpha_{e}}\right\},
$$

where here we made the dependence of $S_{D}$ on $\alpha_{e}$ explicit. Consider an increasing sequence $\alpha_{e}^{i}$ where $i \in \mathbb{N}$ and $\lim _{i \rightarrow \infty} \alpha_{e}^{i} \rightarrow \infty$. This results is a nondecreasing sequence of sets: $\left\{\mathcal{S}_{D}\left(\alpha_{e}^{i}\right)\right\}_{i=1}^{\infty}$ :

$\alpha_{e}^{i}<\alpha_{e}^{i+1} \Rightarrow \frac{\frac{1}{2} \dot{q}^{T} D(q) \dot{q}}{\alpha_{e}^{i}}>\frac{\frac{1}{2} \dot{q}^{T} D(q) \dot{q}}{\alpha_{e}^{i+1}} \Rightarrow \mathcal{S}_{D}\left(\alpha_{e}^{i}\right) \subset \mathcal{S}_{D}\left(\alpha_{e}^{i+1}\right)$. 
As a result:

$\lim _{i \rightarrow \infty} \frac{\frac{1}{2} \dot{q}^{T} D(q) \dot{q}}{\alpha_{e}^{i}}=0 \Rightarrow \lim _{i \rightarrow \infty} \mathcal{S}_{D}\left(\alpha_{e}^{i}\right)=\bigcup_{i \in \mathbb{N}} \mathcal{S}_{D}\left(\alpha_{e}^{i}\right) \supset \operatorname{Int}(\mathcal{S})$,

and $\mathcal{S}_{D}\left(\alpha_{e}^{i}\right) \subset \mathcal{S}$ for all $i \in \mathbb{N}$.

Main result. We now have the necessary constructions to present the main result of this paper-a largely model independent safety-critical controller that ensures the forward invariance of $\mathcal{S}_{D}$ and, therefore, $\mathcal{S}$ in the limit for $\alpha_{e}$ sufficiently large. We will establish this by showing that $h_{D}$ is a valid CBF and that $\dot{h}_{D}$ only depends on the kinematics, the gravity vector $G(q)$, and the inertial matrix $D(q)$. This makes the controller more robust to uncertainty in the dynamics than full model based controllers-which would require knowledge of the Coriolis-centrifugal matrix, $C(q, \dot{q})$.

Theorem 2. Consider a robotic system (10), assumed to be fully actuated with $B$ invertible, and a kinematic safety constraint $h: Q \rightarrow \mathbb{R}$ with corresponding safe set $\mathcal{S}=$ $\left\{(q, \dot{q}) \in Q \times \mathbb{R}^{k}: h(q) \geq 0\right\}$. Let $h_{D}$ be the energy based constraint defined as in (11) with corresponding safe set $\mathcal{S}_{D}$ as given in (12). Then $h_{D}$ is a control barrier function on $\mathcal{S}_{D}$ and given a desired controller $u_{\mathrm{des}}(x, t)$, the following controller for all $(q, \dot{q}) \in \mathcal{S}_{D}$ :

$$
\begin{aligned}
u^{*}(q, \dot{q}, t)=\underset{u \in \mathbb{R}^{m}}{\operatorname{argmin}} & \left\|u-u_{\mathrm{des}}(q, \dot{q}, t)\right\|^{2} \\
\text { s.t. } & \underbrace{-\dot{q}^{T} B u+G(q)^{T} \dot{q}+\alpha_{e} J_{h}(q) \dot{q}}_{\dot{h}_{D}(q, \dot{q}, u)} \geq-\alpha\left(h_{D}(q, \dot{q})\right),
\end{aligned}
$$

guarantees forward invariance of $\mathcal{S}_{D}$, i.e., safety of $\mathcal{S}_{D}$. Additionally, it has a closed form solution:

$u^{*}(x, t)=u_{\mathrm{des}}(q, \dot{q}, t)+ \begin{cases}\frac{B^{T} \dot{q}}{\| B^{T} \dot{\|^{2}}} \Psi\left(x, t ; u_{\mathrm{des}}\right) & \text { if } \Psi\left(x, t ; u_{\mathrm{des}}\right)<0 \\ 0 & \text { if } \Psi\left(x, t ; u_{\mathrm{des}}\right) \geq 0\end{cases}$

where

$\Psi\left(x, t ; u_{\mathrm{des}}\right):=\dot{q}^{T}\left(\alpha_{e} J_{h}(q)^{T}+G(q)-B u_{\mathrm{des}}(x, t)\right)+\alpha\left(h_{D}(q, \dot{q})\right)$.

It is interesting to note that $h_{D}$ is a $\mathrm{CBF}$ on $\mathcal{S}_{D}$ without requiring that $h$ has relative degree 1, i.e., one need not require that $J_{h}(q) \neq 0$ (except on $\partial \mathcal{S}$ ) as in Lemma 2. This reinforces the idea that these energy-based control barrier functions are a natural extension for relative-degree 2 robotic systems.

Proof of Theorem 2. Differentiating $h_{D}$ along solutions yields (and suppressing the dependence on $q$ and $\dot{q}$ ):

$$
\begin{aligned}
\dot{h}_{D} & =-\dot{q}^{T} D \ddot{q}-\frac{1}{2} \dot{q}^{T} \dot{D} \dot{q}+\alpha_{e} J_{h} \dot{q} \\
& =\dot{q}^{T}(C \dot{q}+G-B u)-\frac{1}{2} \dot{q}^{T} \dot{D} \dot{q}+\alpha_{e} J_{h} \dot{q} \\
& =\frac{1}{2} \dot{q}^{T}(-\dot{D}+2 C) \dot{q}-\dot{q}^{T} B u+G^{T} \dot{q}+\alpha_{e} J_{h} \dot{q} \\
& =-\dot{q}^{T} B u+G^{T} \dot{q}+\alpha_{e} J_{h} \dot{q}
\end{aligned}
$$

where the last equality follows from the fact that $\dot{D}-2 C$ is skew symmetric (see [19, Lemma 4.2]). To establish that $h_{D}$ is a CBF, we need only show that (14) has a solution since the inequality constraint in (14) implies that (2) is satisfied in Definition 1. As a result of Lemma 1, the solution to (14) is given by (3). Note that

$L_{f} h_{D}(q, \dot{q})=\left(\alpha_{e} J_{h}(q)+G(q)^{T}\right) \dot{q}, \quad L_{g} h_{D}(q, \dot{q})=-\dot{q}^{T} B$.

Since (3) has a $L_{g} h L_{g} h^{T}$ term in the denominator, to show that (3) is well defined, we need to establish that:

$L_{g} h_{D}(q, \dot{q})=-\dot{q}^{T} B=0 \quad \Rightarrow \quad L_{f} h_{D}(q, \dot{q})+\alpha\left(h_{D}(q, \dot{q})\right) \geq 0$.

Yet $\dot{q}^{T} B=0$ implies that $\dot{q}^{T}=0$ since $B$ is invertible and therefore $L_{f} h_{D}(q, \dot{q})=0$ and since $(q, \dot{q}) \in \mathcal{S}_{D}$ it follows that $h_{D}(q, \dot{q}) \geq 0$ and hence $\alpha\left(h_{D}(q, \dot{q})\right) \geq 0$ implying that (3) is well defined and thus $h_{D}$ is a CBF. Finally, the forward invariance of $\mathcal{S}_{D}$ follows from the results of Lemma 1 and Theorem 1.

Having established Theorem 2, the following corollary demonstrates how to further reduce model dependence.

Corollary 1. Under the conditions of Theorem 2, if there exists $a c_{u}>0$ such that $c_{u} \geq \frac{1}{2} \lambda_{\max }(D(q))$ then replacing the safety constraint (14) in the safety-critical $Q P$ with:

$$
\underbrace{-\dot{q}^{T} B u+G(q)^{T} \dot{q}+\alpha_{e} J_{h}(q) \dot{q}}_{h_{D}(q, \dot{q}, u)} \geq-\alpha\left(-c_{u}\|\dot{q}\|^{2}+\alpha_{e} h(q)\right),
$$

implies safety of $\mathcal{S}_{D}$. Moreover, if in addition $\|G(q)\| \leq c_{u}$, for a large enough $c_{u}>0$ (perhaps larger than previously determined), then the constraint (14) can be replaced by:

$$
\alpha_{e} J_{h}(q) \dot{q}-\dot{q}^{T} B u-c_{u}|\dot{q}| \geq-\alpha\left(-c_{u}\|\dot{q}\|^{2}+\alpha_{e} h(q)\right) .
$$

wherein safety of $\mathcal{S}_{D}$ is guaranteed.

Proof. It can be verified that $-\alpha\left(-c_{u}\|\dot{q}\|^{2}+\alpha_{e} h(q)\right) \geq$ $-\alpha\left(-\frac{1}{2} \lambda_{\max }(D(q))\|\dot{q}\|^{2}+\alpha_{e} h(q)\right) \geq-\alpha\left(h_{D}(q, \dot{q})\right)$, which means that (17) $\Longrightarrow(14)$. The second inequality, (18), follows from the bound on the gravity vector $G$.

Connections with kinematic control. The goal is to now connect the previous constructions with the kinematic controllers defined in Section III. Often, controllers can only be implemented as desired position and velocity commands that are passed to embedded level PD controllers. Moreover, minimizing the difference between the desired and the safe robot velocities often leads to more desirable behaviors with the lower-level commands, which affect the system in much more complex ways. As such, we consider a controller of the form:

$$
u=-K_{\mathrm{vel}}\left(\dot{q}-\dot{q}_{d}^{*}(q, \dot{q}, t)\right)
$$

where $\dot{q}_{d}^{*}(q, t)$ is a desired velocity signal that enforces safety while trying to achieve tracking as in the case of Lemma 2 wherein we have a desired velocity based tracking controller: $\dot{q}_{\mathrm{des}}(q, t):=J_{y}(q)^{\dagger}\left(\dot{x}_{d}(t)-\lambda\left(y(q)-x_{d}(t)\right)\right)$ for $\lambda>0$. The following is a result of the direct application of Theorem 2 in the context of the controller (19). 


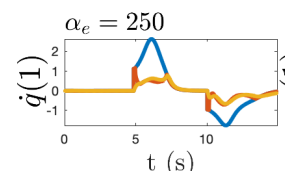

$\mathrm{t}(\mathrm{s})$
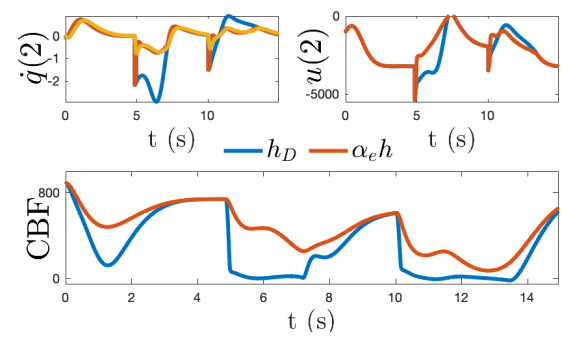

Fig. 3. Energy-based kinematic CBF on the 6 DOF manipulator. Safety is guaranteed regardless of the choice of $\alpha_{e}$, but performance improves as $\alpha_{e}$ increases. The times taken to complete the second portion of the task, near the obstacle, are 10.07, 8.06, and 6.86 sec for $\alpha_{e}=\{250,500,1500\}$, compared to values of 7.60 to 7.94 for the purely kinematic case and 5.79 for CBF-free case. See [1] for the video.

Theorem 3. Consider a robotic system (10), and assume it is fully actuated. Given a kinematic safety constraint $h: Q \rightarrow \mathbb{R}$ and the associated dynamically consistent extended $C B F h_{D}$ : $Q \times \mathbb{R} \rightarrow \mathbb{R}$ as given in (11) with associated safe set $\mathcal{S}_{D}$, along with a desired trajectory $x_{d}(t)$ in the task space $x=y(q)$. The $D$ controller (19) with $K_{\mathrm{vel}} \succ 0$ and the following $Q P$ :

$$
\begin{aligned}
\dot{q}_{d}^{*}=\underset{\dot{q}_{d} \in \mathbb{R}^{n}}{\operatorname{argmin}}\|\dot{q}_{d}-\overbrace{J_{y}^{\dagger}\left(\dot{x}_{d}-\lambda\left(y-x_{d}\right)\right)}^{\dot{q}_{\mathrm{des}}(q, t)}\|^{2} & \\
\text { s.t. } & \underbrace{\alpha_{e} J_{h} \dot{q}+\dot{q}^{T} B K_{\mathrm{vel}} \dot{q}-\dot{q}^{T} B K_{\mathrm{vel}} \dot{q}_{d}+G^{T} \dot{q}}_{\dot{h}_{D}\left(q, \dot{q}, \dot{q}_{d}\right)} \geq-\alpha\left(h_{D}\right),
\end{aligned}
$$

guarantees forward invariance, i.e., safety, of $\mathcal{S}_{D}$. Moreover, it has a closed form solution:

$\dot{q}_{d}^{*}=\dot{q}_{\mathrm{des}}+ \begin{cases}\frac{K_{\text {vel }}^{T} B^{T} \dot{q}}{\left\|K_{\mathrm{vel}}^{T} B^{T} \dot{q}\right\|^{2}} \Psi\left(q, \dot{q}, t ; q_{\mathrm{des}}\right) & \text { if } \Psi\left(q, \dot{q}, t ; q_{\mathrm{des}}\right)<0 \\ 0 & \text { if } \Psi\left(q, \dot{q}, t ; q_{\mathrm{des}}\right) \geq 0\end{cases}$

where

$\Psi\left(q, \dot{q}, t ; \dot{q}_{\mathrm{des}}\right):=\dot{q}^{T}\left(\alpha_{e} J_{h}^{T}+B K_{\mathrm{vel}} \dot{q}-B K_{\mathrm{vel}} \dot{q}_{\mathrm{des}}+G\right)+\alpha\left(h_{D}\right)$.

Proof of Theorem 3 is omitted as it is a straightforward extension of Theorem 2. It may be the case, as with industrial actuators, that $K_{\mathrm{vel}}$ is not known. In that case, it can typically be determined from experimental data. Formally, one can guarantee safety by utilizing adaptive control barrier functions [20]. Similar to Remark 1, we can reformulate the constraints to eliminate the $D$ and $G$ matrices to yield robust QPs.

Example 2 (Energy-based kinematic CBF). The 6 DOF manipulator from Example 1 is now filtered with the constraint given in (17), using $c_{u}=5 \lambda_{\max }(D)$. Figure 3 shows the result for different values of $\alpha_{e}$. Safety is guaranteed regardless of the value of $\alpha_{e}$, but as the value increases, the manipulator is able to move faster and get closer to obstacles, resulting in better performance. Each input was computed in under $10 \mu \mathrm{s}$.

\section{UNDERACTUATED SYSTEMS}

The methods developed can also be applied to underactuated systems, i.e., where $m \leq k$ and we have a potentially non-singular actuation matrix $B$. The key idea is to treat $h(q)$ as one of the coordinates. Choose a mapping $\Phi(q):=(w(q), h(q))$, where $w$ is chosen such that $\Phi$ is a diffeomorphism. This can be easily obtained for non-singular configurations. We obtain the derivative as

$$
\left[\begin{array}{c}
\dot{w}(q, \dot{q}) \\
\dot{h}(q, \dot{q})
\end{array}\right]=J_{e}(q) \dot{q}
$$

where $J_{e}(q)$ is the Jacobian matrix. $J_{e}$ is non-singular by property of diffeomorphism. We re-write the equations of motion of the robot as

$$
D_{e}(q)\left[\begin{array}{l}
\ddot{w} \\
\ddot{h}
\end{array}\right]+C_{e}(q, \dot{q})\left[\begin{array}{l}
\dot{w} \\
\dot{h}
\end{array}\right]+G_{e}(q)=J_{e}(q)^{-T} B u,
$$

where

$$
\begin{aligned}
D_{e}(q) & =J_{e}(q)^{-T} D(q) J_{e}(q)^{-1} \\
C_{e}(q, \dot{q}) & =J_{e}(q)^{-T} C(q) J_{e}(q)^{-1}+J_{e}(q)^{-T} D(q) \dot{J}_{e}(q)^{-1} \\
G_{e}(q) & =J_{e}(q)^{-T} G(q),
\end{aligned}
$$

are the new terms that define the dynamics in the transformed space. It can be verified that the properties of $D_{e}, C_{e}$ will be same as that of $D, C$, i.e., $D_{e}$ is symmetric positive definite, and $\dot{D}_{e}-2 C_{e}$ is skew-symmetric. More details are in [19, Chapter 4, Section 5.4]. We can separate (23) into two parts:

$$
\begin{aligned}
& D_{11}(q) \ddot{w}+D_{12}(q) \ddot{h}+C_{1}(q, \dot{q}) \dot{q}+G_{1}(q)=B_{1}(q) u \\
& D_{21}(q) \ddot{w}+D_{22}(q) \ddot{h}+C_{2}(q, \dot{q}) \dot{q}+G_{2}(q)=B_{2}(q) u,
\end{aligned}
$$

where the terms corresponding to $D, C, G, B$ are apparent from the setup. $\ddot{w}$ can be eliminated from (25) to obtain

$\underbrace{\left(D_{22}-D_{21} D_{11}^{-1} D_{12}\right)}_{D_{h}} \ddot{h}+\underbrace{\left(C_{2}-D_{21} D_{11}^{-1} C_{1}\right)}_{C_{h}} \dot{q}+\underbrace{G_{2}-D_{21} D_{11}^{-1} G_{1}}_{G_{h}}=\underbrace{\left(B_{2}-D_{21} D_{11}^{-1} B_{1}\right)}_{B_{h}} u$,

where $D_{h}$ is nothing but the Schur complement form, and it is known to be symmetric positive definite [21, Proposition 1]. Note that here $B_{h}: Q \rightarrow \mathbb{R}^{1 \times m}$ is the mapping from $u$ to the joints, which is assumed to have full row rank (in other words, $h$ is assumed to be inertially coupled with $u$. This may not be satisfied for all $Q$, in which case a subset $Q_{u} \subset Q$ is chosen (for example, in the cart-pole, pole-angle is not inertially coupled with $u$ when it is horizontal). With this formulation, we have the following theorem. 


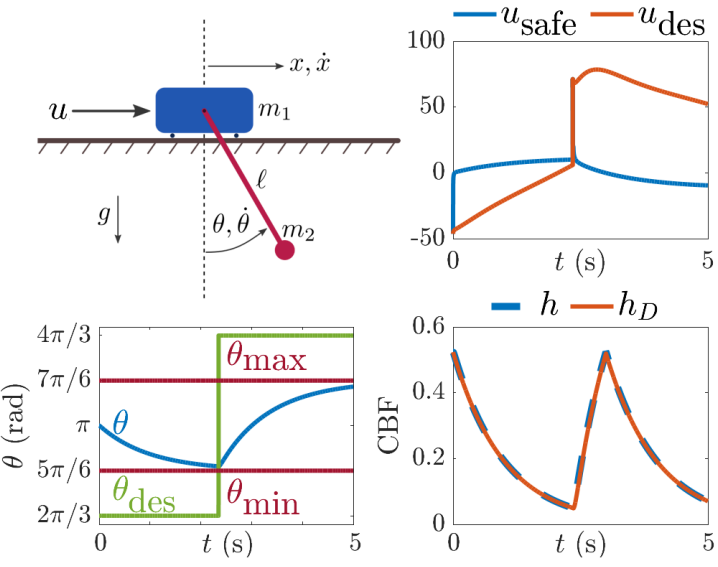

Fig. 4. Cart-pole system with energy-based CBF.

Theorem 4. Consider a robotic system (10) and a kinematic safety constraint: $h: Q \rightarrow \mathbb{R}$. Consider the dynamically consistent extended CBF for underactuated systems:

$$
\widehat{h}_{D}(q, \dot{q}):=-\frac{1}{2} \dot{h}(q, \dot{q})^{T} D_{h}(q) \dot{h}(q, \dot{q})+\alpha_{e} h(q)
$$

with the safe set: $\widehat{S}_{D}:=\left\{(q, \dot{q}) \in Q \times \mathbb{R}^{k}: \widehat{h}_{D}(q, \dot{q}) \geq 0\right\}$. Then $\widehat{S}_{D} \subset \mathcal{S}$ and for all $(q, \dot{q}) \in \widehat{S}_{D}$ the following controller:

$$
\begin{aligned}
& u^{*}(q, \dot{q}, t)=\underset{u \in \mathbb{R}^{m}}{\operatorname{argmin}}\left\|u-u_{\mathrm{des}}(q, \dot{q}, t)\right\|^{2} \\
& \text { s.t. }-\frac{1}{2} \dot{h}_{h} \dot{h}-\dot{h}\left(-C_{h} \dot{q}-G_{h}\right)+\alpha_{e} \dot{h}-\dot{h} B_{h} u \geq-\alpha\left(\widehat{h}_{D}(q, \dot{q})\right)
\end{aligned}
$$

guarantees forward invariance of $\widehat{S}_{D}$, i.e., safety of $\widehat{S}_{D}$.

Proof. Differentiating $\widehat{h}$ yields:

$$
\dot{\hat{h}}_{D}=-\frac{1}{2} \dot{h}_{h} \dot{h}-\dot{h}\left(-C_{h} \dot{q}-G_{h}\right)+\alpha_{e} \dot{h}-\dot{h} B_{h} u .
$$

It can be verified that if $\dot{h}=0$, then the inequality in (28) is satisfied. The safety property follows directly.

Remark 1. Similar to Corollary 1, we can eliminate some of the model-based terms in (28). Specifically, we can replace the constraint in the QP with the following:

$$
-\frac{1}{2} c_{l} \dot{h}^{2}-c_{u}|\dot{h}|\left(|\dot{q}|^{2}+1\right)+\alpha_{e} \dot{h}-\dot{h} B_{h} u \geq-\alpha\left(-c_{u} \dot{h}^{2}+\alpha_{e} h(x)\right) \text {, }
$$

where $c_{l}, c_{u}$ are constants that bound the norms: $c_{l} \leq\left\|D_{h}\right\| \leq$ $c_{u},\left\|C_{h}\right\| \leq c_{u}|\dot{q}|,\left\|G_{h}\right\| \leq c_{u}$. We have used the same notations for convenience. Note that these bounds may not exist for all $(q, \dot{q}) \in Q \times \mathbb{R}^{k}$, and they are dependent on the validity of the coordinate transformation $\Phi$. This is usually avoided by choosing a smaller configuration set $Q_{u}$. More details on the bounds are in [21].

Example 3 (Cart-Pole System). To demonstrate these concepts, we consider the cart-pole system with two states, the cart position $x$ and the pole angle $\theta$. The system is actuated through a force input $u$ applied to the cart, which moves freely in a line. The safety constraint is to ensure that pole remains mostly upright, with $\theta \in\left[\frac{5 \pi}{6}, \frac{7 \pi}{6}\right]$. We choose $w=x$ and $h=\left(\frac{\pi}{6}\right)^{2}-(\theta-\pi)^{2}$. The results of applying the QP (28) are shown in Figure 4.

\section{CONCLUSIONS}

We demonstrated how to guarantee safety-critical kinematic constraints via control barrier functions. We first introduced a purely kinematic $\mathrm{CBF}$, and then extended this formulation to construct a new form of CBF constraints, which incorporates kinetic energy to minimize model dependence. We show how this can be implemented at the velocity or torque level. Simulation results show that the resulting CBF-QPs guarantee safety for both underactuated and fully-actuated systems. Future work will focus on guaranteeing safety under perturbations, system uncertainties, and incorporation of multiple constraints.

\section{REFERENCES}

[1] "Simulation video." http://youtube.com/watch?v=ilLRA7fpVBI

[2] B. Siciliano, "Kinematic control of redundant robot manipulators: A tutorial," Journal of intelligent and robotic systems, vol. 3, no. 3, pp. 201-212, 1990.

[3] J. Xiang, C. Zhong, and W. Wei, "General-weighted least-norm control for redundant manipulators," IEEE Transactions on Robotics, vol. 26, no. 4 , pp. 660-669, 2010

[4] G. Antonelli and S. Chiaverini, "Kinematic control of platoons of autonomous vehicles," IEEE Transactions on Robotics, vol. 22, no. 6, pp. 1285-1292, 2006.

[5] O. Khatib, "Real-time obstacle avoidance for manipulators and mobile robots," in Autonomous robot vehicles. Springer, 1986, pp. 396-404.

[6] B. Faverjon and P. Tournassoud, "A local based approach for path planning of manipulators with a high number of degrees of freedom," in Proceedings. 1987 IEEE International Conference on Robotics and Automation, vol. 4, 1987, pp. 1152-1159.

[7] O. Kanoun, F. Lamiraux, and P. Wieber, "Kinematic control of redundant manipulators: Generalizing the task-priority framework to inequality task," IEEE Transactions on Robotics, vol. 27, no. 4, pp. 785-792, 2011.

[8] A. Ames, X. Xu, J. Grizzle, and P. Tabuada, "Control barrier function based quadratic programs for safety critical systems," IEEE Trans. Automatic Control, vol. 62, p. 3861-3876, 2017.

[9] M. Jankovic, "Robust control barrier functions for constrained stabilization of nonlinear systems," Automatica, vol. 96, pp. 359-367, 2018.

[10] W. S. Cortez, D. Oetomo, C. Manzie, and P. Choong, "Control barrier functions for mechanical systems: Theory and application to robotic grasping," IEEE Transactions on Control Systems Technology, 2019.

[11] M. Rauscher, M. Kimmel, and S. Hirche, "Constrained robot control using control barrier functions," in 2016 IEEE/RSJ International Conference on IROS. IEEE, 2016, pp. 279-285.

[12] L. Wang, A. Ames, and M. Egerstedt, "Safety barrier certificates for collisions-free multi-robot systems," IEEE Transactions on Robotics, vol. 33, no. 3, pp. 661-674, 2017.

[13] L. Lindemann and D. V. Dimarogonas, "Control barrier functions for multi-agent systems under conflicting local signal temporal logic tasks," IEEE Control Systems Letters, vol. 3, no. 3, pp. 757-762, 2019.

[14] A. D. Ames, S. Coogan, M. Egerstedt, G. Notomista, K. Sreenath, and P. Tabuada, "Control barrier functions: Theory and applications," in 2019 18th European Control Conference (ECC). IEEE, 2019, pp. 3420-3431.

[15] C. T. Landi, F. Ferraguti, S. Costi, M. Bonfè, and C. Secchi, "Safety barrier functions for human-robot interaction with industrial manipulators," in 2019 18th ECC. IEEE, 2019, pp. 2565-2570.

[16] S. Kolathaya, "Energy based Control Barrier Functions for Robotic Systems," August 2020. [Online]. Available: https://www.techrxiv.org/articles/preprint/Energy_based_Control_ Barrier_Functions_for_Robotic_Systems/12831503

[17] X. Xu, P. Tabuada, J. W. Grizzle, and A. D. Ames, "Robustness of control barrier functions for safety critical control," IFAC-PapersOnLine, vol. 48 , no. 27 , pp. $54-61,2015$.

[18] Q. Nguyen and K. Sreenath, "Exponential control barrier functions for enforcing high relative-degree safety-critical constraints," in 2016 American Control Conference (ACC). IEEE, 2016, pp. 322-328.

[19] R. M. Murray, Z. Li, S. S. Sastry, and S. S. Sastry, A mathematical introduction to robotic manipulation. CRC press, 1994.

[20] A. J. Taylor and A. D. Ames, "Adaptive safety with control barrier functions," in 2020 American Control Conference (ACC), 2020, pp. $1399-1405$

[21] S. Kolathaya, "Local stability of PD controlled bipedal walking robots," Automatica, vol. 114, p. 108841, 2020. 